\title{
Type I Interferons
}

Theresa K. Resch ${ }^{\mathrm{a}}$, Gabriele Reichmann ${ }^{\mathrm{b}}$ and Zoe Waibler ${ }^{\mathrm{a} *}$

a Junior Research Group “Novel Vaccination Strategies and Early Immune Responses”, Paul-Ehrlich-Institut, Langen, Germany

${ }^{\mathrm{b}}$ Department of Immunology, Paul-Ehrlich-Institut, Langen, Germany

\section{Synonyms}

Interferon- $\alpha$ /interferon- $\beta$; Type I interferons

\section{Definition}

Type I interferons (IFNs) were identified more than 50 years ago by Isaacs and Lindenmann as a soluble factor that can interfere with viral replication and is able to block infection. Meanwhile, type I IFNs are broadly recognized for their antiviral, antitumoral, and immunomodulatory capacities. Even though virtually any cell type is able to produce type I IFNs, they are secreted mainly by plasmacytoid dendritic cells (pDC). The family of type I IFNs consists of 12 different IFN- $\alpha$ subtypes in man (13 subtypes in the mouse) and one subtype each of IFN- $\beta$, IFN- $\omega$, IFN- $\varepsilon$, and IFN- $\kappa$. IFN- $\delta$ is expressed in pigs only, IFN- $\zeta$ in rodents, and IFN- $\tau$ in ruminants (Yoneyama and Fujita 2010).

\section{Biosynthesis and Release}

Upon appropriate stimulation of a host, cells respond within hours with the secretion of type I IFN. Hence, type I IFNs are described as first line of defense upon infections. The induction of type I IFNs is initiated via the detection of pathogen-associated molecular patterns (PAMPs), such as derived from viruses and bacteria, or danger-associated molecular patterns (DAMPs), such as components associated with damage or malfunction of host cells. Toll-like receptors (TLRs) are the most prominent pattern recognition receptors (PRRs) either localized at the cell surface or at the endosomal membrane. Cytosolic PRRs include members of the NOD-like receptor (NLR) family and members of the retinoic acid-inducible gene I (RIG-I)-like receptor (RLR) family (RIG-I and melanoma differentiation-associated gene 5 (MDA5)) (Pitha 2007; Yoneyama and Fujita 2010).

The signal transduction after ligation of PAMPs to their respective PRR is mediated by different adaptor molecules resulting in specific cellular responses. TLR adaptor molecules include the myeloid differentiation primary response gene (88) (MyD88), TIR-domain-containing adapter-inducing interferon- $\beta$ (TRIF, also called TICAM1), and TRIF-related adapter molecule (TRAM). RLRs recruit the mitochondrial antiviral-signaling protein (MAVS, also known as VISA, Cardif, or IPS-1) located at the outer membrane of mitochondria (Yoneyama and Fujita 2010). Downstream of those different adaptor molecules, multi-protein complexes are formed containing IRF3, NF- $\mathrm{B}$, or ATF-2/cJun. These complexes are translocated to the nucleus leading to the induction of type I IFNs and other pro-inflammatory cytokines. After secretion, type I IFNs bind to their cellular receptor, the type I IFN receptor (IFNAR), in an auto and paracrine manner. The IFNAR is a heterodimeric transmembrane receptor consisting of an IFNAR1 and

\footnotetext{
*Email: zoe.waibler@pei.de
} 
an IFNAR2 chain, expressed on almost all nucleated cells. Binding leads to the induction of the JAK/STAT signaling pathway resulting in an enhanced production of all type I IFN subtypes as well as the regulation of interferon-stimulated genes (ISGs) (Pitha 2007).

\section{Biological Activities}

Up to now, more than 2000 ISGs have been identified (de Weerd et al. 2013). Classical antiviral ISGs include the cytoplasmic protein kinase R (PKR), the $2^{\prime}-5^{\prime}$-oligoadenylate synthetase (OAS), and Mx GTPases. PKR is activated by double-stranded (ds)RNA as derived from viral replication, and phosphorylates eukaryotic initiation factor 2 (eIF2 $\alpha$ ), preventing translation of (viral) RNA. Upon binding of dsRNA, OAS initiates synthesis of polymeric ATP whose only known function is to activate latent RNase L to degrade (viral) RNA. In addition, disruption of the RNA machinery can lead to apoptosis of the infected cell. Moreover, cleaved RNA fragments can bind to cytosolic RNA sensors such as RIG-I or MDA5 leading to an enhanced type I IFN production. In man, two Mx proteins, MxA and MxB, are induced by type I IFNs. The antiviral function of these proteins is not fully understood yet, but it could be shown that Mx GTPases interfere with viral trafficking and activation of viral polymerases (Pitha 2007).

In addition to direct anti-pathogenic effects via the induction of ISGs, type I IFNs impact adaptive immune cell functions and hence act at the interface between innate and adaptive immunity. Direct effects of type I IFNs on T-cell expansion and effector functions have been demonstrated (Frenz et al. 2010), and additionally, type I IFNs have been reported to shape antibody responses by enhancing the humoral immune response and promoting isotype switching (Le Bon et al. 2001).

\section{Pathophysiological Relevance and Modulation by Drugs}

Having the pleiotropic antiviral effects in mind, it is no surprise that type I IFNs were licensed for the treatment of severe acute viral infections such as SARS and are, along with Ribavirin, currently the means of choice for the treatment of chronic hepatitis B/C virus infections. Type I IFNs are also extensively used for the treatment of several types of cancer including hematological malignancies (e.g., hairy cell leukemia, follicular lymphoma, cutaneous T-cell lymphoma) and solid tumors (e.g., melanoma, renal cell carcinoma, Kaposi's sarcoma). Besides having a direct antiproliferative activity on tumor cells (i.e., induction of cell cycle arrest or apoptosis), type I IFNs inhibit angiogenesis and can act as an adjuvant in cancer vaccines.

Given the potency of type I IFNs as antiviral and immune-activating cytokines, their expression has to be regulated tightly in order to avoid severe cell damage. Hence, it is not surprising that deregulated type I IFN expression has been associated with a number of diseases characterized by uncontrolled inflammatory processes.

Systemic lupus erythematosus (SLE) is an inflammatory autoimmune disorder affecting the skin, kidney, musculoskeletal, and hematologic systems. Several lines of evidences indicate a central role for IFN- $\alpha$ in the pathogenesis of SLE. It has been shown that $70-80 \%$ of adult patients and close to $100 \%$ of pediatric patients overexpress type I IFN-induced genes (IFN signature) in both blood cells and peripheral tissues. The level of type I IFN target gene expression correlates with disease severity and activity. In line with this, children usually show a more aggressive course of disease than adult patients. In addition to an IFN signature, SLE patients have elevated serum type I IFN levels, particularly those patients with higher disease activity. Interestingly, several studies report the presence of anti-IFN- $\alpha$ antibodies in sera of SLE patients. The origin and mechanisms of induction of these autoantibodies are not clear yet. However, the 
majority of SLE patients positive for anti-IFN- $\alpha$ antibodies show significantly lower levels of serum type I IFN bioactivity, a reduced IFN signature, and lower disease activity (Lauwerys et al. 2013).

Another line of evidence indicating the central role for IFN- $\alpha$ in the pathology of SLE comes from longterm type I IFN-treated patients with malignant or viral diseases. The continuous administration of IFN- $\alpha$ to these patients can induce lupus-like symptoms indistinguishable from spontaneous SLE. Interestingly, those lupus-like symptoms were completely resolved when type I IFN administration was discontinued (Niewold and Swedler 2005).

Experimental evidences from murine models further underline the central role of type I IFNs for the etiopathology of SLE. Type I IFNs accelerate the SLE-like syndrome in lupus-prone mice, and deletion of the IFNAR protects those animals (Agrawal et al. 2009). In line with this, mice deficient for SOCS (suppressor of cytokine signaling proteins), involved in inhibiting the JAK/STAT signaling pathway, develop the SLE-like syndrome (Banchereau and Pascual 2006). Also, mice deficient for negative regulators of TLR signaling spontaneously develop inflammatory disorders by aberrant type I IFN production (Marshak-Rothstein 2006).

Multiple mechanisms can lead to the uncontrolled type I IFN induction in SLE. Most probably, a combination of viral infection(s) and a (genetic) predisposition of the patient such as genetic variations in TLR pathway components, IRFs, or RLR pathway components culminate in aberrant type I IFN secretion. Those elevated IFN- $\alpha$ levels contribute to SLE. Type I IFNs were shown to induce differentiation of monocytes into DC, maturation of DC, and priming of antigen-presenting cells. Furthermore, they improve T-cell-mediated responses to (auto)antigens. With respect to (auto)antibody production, type I IFNs were shown to promote strong IgG secretion and to potentiate signaling through the B-cell receptor (Frenz et al. 2010; Lauwerys et al. 2013; Waibler et al. 2009). Hence, the various modes of action of type I IFNs, which are beneficial in an antiviral immune response (see above), are deleterious in the etiopathology of SLE.

Given the prominent role of type I IFNs for the development of SLE, a number of clinical trials targeting the type I IFN system are ongoing in SLE patients. Two IFN- $\alpha$ neutralizing monoclonal antibodies (mAbs) are tested, namely, Sifalimumab and Rontalizumab, one mAb blocking the IFNAR (MEDI-546), and one IFN- $\alpha$ vaccine (IFN- $\alpha$ kinoid) aiming at the transient induction of polyclonal IFN- $\alpha$ neutralizing antibodies (summarized in Table 1). Due to the early stage of development of all therapeutics (primarily phase II), conclusions on efficacy and safety of the treatment are limited yet. For Sifalimumab, a dose-dependent decrease in the expression of type I IFN-induced genes was reported $(n=33)$ when compared to the placebo group $(n=17)$ and a trend toward disease improvement could be observed. For Rontalizumab, in a subset of all treated patients $(n=48)$, reduction of the IFN signature was documented as well (Lauwerys et al. 2013). Whether one of the abovementioned therapeutics will prove beneficial for SLE patients will need to be demonstrated in phase III trials.

Another example for type I IFNs as central mediators of inflammation is psoriasis. Here, keratinocytes proliferate abnormally in response to a chronic inflammatory reaction. In particular at an early stage of disease, $\mathrm{pDC}$ are recruited to pre-plaque areas, where they become activated and secrete large quantities of

Table 1 Anti-type I IFN therapeutic concepts in clinical trials

\begin{tabular}{|c|c|c|c|c|}
\hline Target & Therapeutic concept & Product & Indication & Stage of clinical trial \\
\hline \multirow[t]{2}{*}{ IFN- $\alpha$} & \multirow[t]{2}{*}{ Neutralizing mAb } & \multirow[t]{2}{*}{ Sifalimumab (MEDI-545) } & SLE & Phase II \\
\hline & & & Psoriasis & Phase I \\
\hline IFN- $\alpha$ & Neutralizing $\mathrm{mAb}$ & Rontalizumab & SLE & Phase II \\
\hline IFNAR & Blocking mAb & MEDI-546 & SLE & Phase II \\
\hline IFN- $\alpha$ & Vaccine & IFN- $\alpha$ kinoid & SLE & Phase I-II \\
\hline
\end{tabular}

All trials in this table are listed in the ClinicalTrials.gov registry 
type I IFNs (Nestle et al. 2005). Consequently, as reported for SLE, an IFN signature is induced in keratinocytes within psoriatic plaques (van der Fits et al. 2004). What exactly drives pDC activation in this early disease stage is not clear yet, but the involvement of TLR ligands has been postulated (Nestle et al. 2005). In a xenograft mouse model of human psoriasis, Nestle et al. demonstrated that blocking the IFNAR or inhibiting the ability of $\mathrm{pDC}$ to produce IFN- $\alpha$ prevented the development of psoriasis (Nestle et al. 2005). In line with this, it has been shown that continuous excessive type I IFN signaling in IRF2deficient mice causes an inflammatory skin disease resembling psoriasis (Hida et al. 2000).

As for SLE, reports indicate an association between the developments of psoriatic eruptions in hepatitis $\mathrm{C}$ patients while receiving IFN- $\alpha$ therapy. Of note, cessation of the IFN- $\alpha$ therapy led to resolution of psoriatic-like symptoms in $93 \%$ of the cases (Afshar et al. 2013).

Given the central role of type I IFNs in psoriasis, a clinical trial has been conducted to evaluate the safety and tolerability of single-dose IFN- $\alpha$-neutralizing Sifalimumab application in adult patients with chronic plaque psoriasis. However, the phase I clinical trial indicated no significant inhibition of the type I IFN signature and no clinical activity of the mAb (Bissonnette et al. 2010). This might be related to the fact that only initial stages of disease development seem to be type I IFN-dependent, whereas late (chronic) stages are rather depending on the activity of tumor necrosis factor (Banchereau and Pascual 2006).

In addition to SLE and psoriasis, there are a number of experimental models and inflammatory disorders for which a central role of type I IFNs in etiopathology has been proposed. In Sjögren's syndrome, foci of inflammation in salivary glands are positive for IFN- $\alpha$, serum type I IFN levels are elevated, and affected organs show an IFN signature. Elevated type I IFN levels and/or an IFN signature were also prominent in dermatomyositis, Aicardi-Goutières syndrome, Cree encephalitis, and type 1 diabetes (Bronson et al. 2012). In a murine model, the development of Lyme arthritis after Borrelia burgdorferi infection is associated with an IFN signature in affected joints and blocking type I IFN signaling reduced both the IFN signature and the development of arthritis (Miller et al. 2008).

Collectively, data derived from both experimental animal models and clinical investigations pinpoint toward a crucial role of type I IFNs in numerous inflammatory diseases and underline the central role of type I IFNs as mediators of inflammation. However, type I IFNs also mediate anti-inflammatory effects, although the data regarding the anti-inflammatory and disease-limiting capacity of treatment with type I IFNs are rather inconsistent and distinct roles for IFN- $\alpha$ and IFN- $\beta$ have been proposed.

The most prominent example for the anti-inflammatory capacity of type I IFNs is IFN- $\beta$ treatment of patients suffering from multiple sclerosis (MS), an inflammatory demyelinating disease of the central nervous system (CNS). Long-term treatment with IFN- $\beta$ of patients with relapsing-remitting MS attenuates the course and severity of the disease and can reduce the frequency of relapses in about one third of patients (McCormack and Scott 2004). The precise mechanism of action of IFN- $\beta$ is still elusive, but it seems to act immunomodulatory at various set points of the disease. MS can be mediated by Th1 and Th17 cells, respectively. IFN- $\beta$ can induce the production of IL-27 which is a potent inhibitor of Th17 cells, and in general, type I IFNs can reduce the number of effector T cells. Moreover, upon IFN- $\beta$ treatment, leucocytes are retained within the lymph nodes and hence do not infiltrate the CNS. In addition, IFN- $\beta$ treatment of microglia cells reduces their ability to present antigens, limiting the risk of being detected and eliminated by cytotoxic T cells or NK cells (Yong et al. 1998).

Even though IFN- $\beta$ can be a powerful tool in treatment of MS, 10-50\% of relapsing-remitting MS patients do not respond to treatment (Rio et al. 2006). In some patients, treatment with IFN- $\beta$ actually induces exacerbations of the disease (Shimizu et al. 2008). In line with this, acute MS-like demyelinating disease with extensive lesions throughout the brain and the thoracic spinal cord has been reported secondary to IFN- $\alpha$ treatment of hepatitis C patients (Matsuo and Takabatake 2002). 
Rheumatoid arthritis (RA) is a chronic inflammatory disorder mainly characterized by the progressive damage of synovial joints and variable extra-articular manifestations. Several observations indicate an inflammatory role for type I IFN in RA. First, as for SLE and other indications (see above), an IFN signature could be detected in a subset of RA patients. Second, RA patients collectively display increased plasma type I IFN activity relative to levels in healthy controls. Finally, IFN- $\beta$ is present in RA synovial membranes. However, IFN- $\beta$ reduces synoviocyte proliferation in vitro which was considered as an antiinflammatory feature. Anti-inflammatory and disease-limiting properties of type I IFNs were also demonstrated in a collagen-induced arthritis model and an adjuvant arthritis model. Here, the injection of IFN- $\beta$ resulted in reduced disease activity and the inhibition of cartilage and bone destruction. Of note, independent clinical trials indicated that IFN- $\beta$ is not effective in RA with regard to clinical or radiographic scores (Axtell et al. 2011; Crow 2010).

Inflammatory bowel disease (IBD) is an intestinal inflammation with two major clinical entities, namely, Crohn's disease and ulcerative colitis (UC). Most likely, IBD results from a disturbed interplay between the intestinal mucosa and gut microbiota. Experimental data indicated a beneficial role for type I IFN in limiting disease. First, it was shown that TLR3 and TLR9 ligand-induced production of type I IFNs reduced the severity of colonic injury and inflammation in murine models of experimental colitis and neutralizing type I IFN abolished this beneficial effect. Next, compared to their wild-type counterparts, IFNAR-deficient mice are highly susceptible to dextran sulfate sodium (DSS)-induced colitis. Finally, administration of IFN- $\beta$ to DSS-treated mice decreased the disease activity and the histological scores of DSS-treated mice.

Clinical data from type I IFN-treated IBD patients are highly variable. While some studies indicated beneficial effects of IFN- $\alpha$ and IFN- $\beta$ treatment of UC patients, other studies failed to demonstrate disease-limiting capacities of this treatment. Moreover, cases are reported of exacerbation of UC during the course of IFN- $\alpha$ therapy of hepatitis $C$ virus infection and induction of UC in the context of IFN- $\beta$ treatment of MS (Gonzalez-Navajas et al. 2012; Rauch et al. 2013).

Taken together, type I IFNs are potent pleiotropic cytokines involved in numerous immunological processes. They are licensed for treatment in several indications including viral infections, cancer, and MS. Given the pro-inflammatory role of type I IFNs in SLE and psoriasis, clinical trials were initiated and are ongoing, respectively, aiming at inhibiting type I IFN signaling. We summarized some (but by no means all) experimental and clinical evidence indicating highly ambivalent and even contrary effects upon both exogenous application of type I IFNs and the inhibition of type I IFN signaling. Currently, no reliable biomarkers are available predicting the outcome of (anti) type I IFN treatment.

Several attempts are discussed aiming at resolving this conundrum. Most authors agree on the distinct properties of type I IFN subtypes, particularly of IFN- $\alpha$ subtypes vs. IFN- $\beta$, in terms of binding to the IFNAR and subsequent target gene expression. Indeed, binding capacities of type I IFN subtypes to the IFNAR can vary (Crow 2010). Moreover, de Weerd et al. recently identified a unique IFN- $\beta /$ IFNAR1 complex that signals independently of the IFNAR 2 chain and induces a distinct and IFN- $\beta$-specific set of genes and functional responses (de Weerd et al. 2013). Whether this IFN- $\beta$ /IFNAR1 signaling is involved in inflammatory disorders may be a matter of future investigations.

Also the severity of inflammation and the overall cytokine milieu prior to type I IFN treatment may decide between adverse or protective effects of the treatment (Axtell et al. 2011; Rauch et al. 2013). The discrepant roles of type I IFNs in two different murine sepsis models and a DSS colitis model, varying in the severity of the inflammatory response, pinpoint toward this direction (Rauch et al. 2013).

Axtell et al. argue that the $\mathrm{T}$ helper cell subset involved in the etiology of the respective disease determines the outcome of a type I IFN treatment: IFN- $\beta$ shall inhibit symptoms in diseases with a Th1 bias but promote pathology in diseases with a Th17 bias (Axtell et al. 2011). 


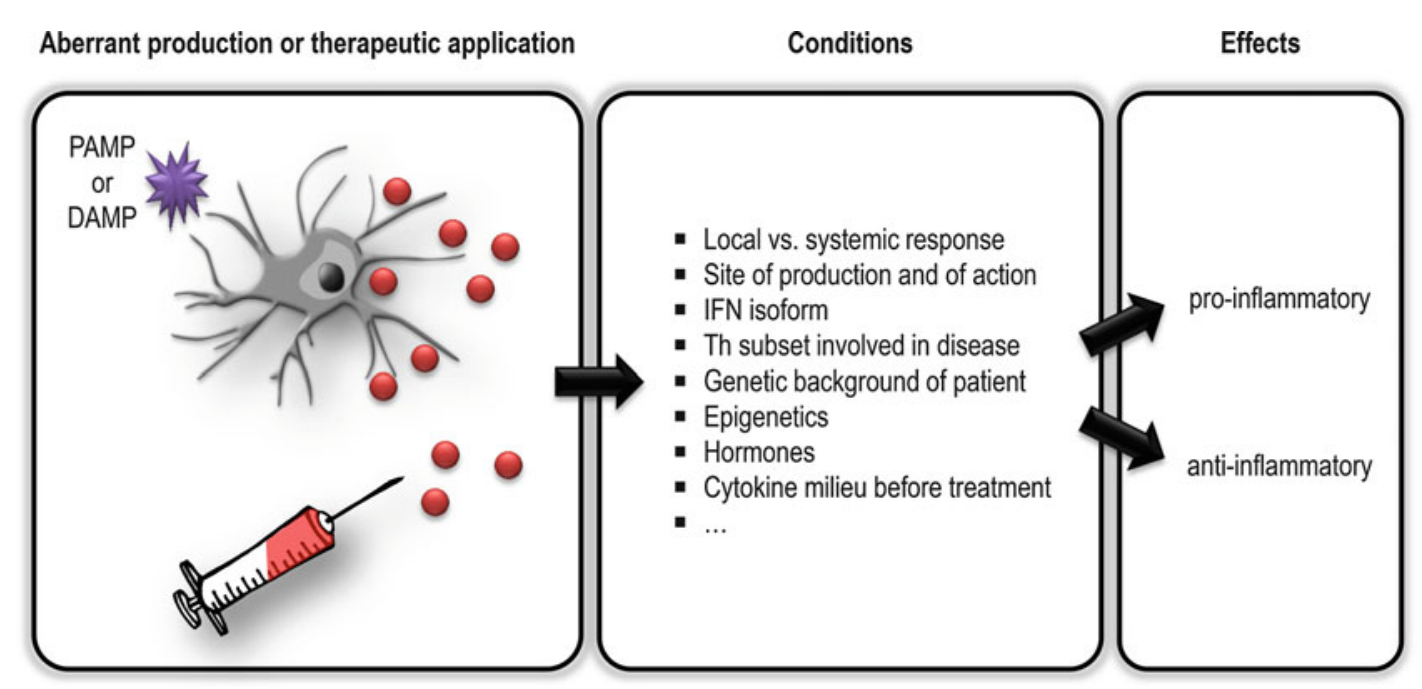

Fig. 1 Pro- and anti-inflammatory effects of type I IFNs. Aberrant production or therapeutic application of type I IFNs can mediate pro- or anti-inflammatory effects depending on patient-specific condition

Without doubt, the genetic predisposition of a patient has a major impact in the onset and cause of an inflammatory disease. For SLE, multiple genetic loci associated with disease susceptibility have been described. Particularly, cytokine-induced signaling components seem to be affected (e.g., Kariuki et al. 2010). Exemplarily, genetic variations in genes encoding for TLR7, IRFs, MDA5, and IPS-1 have been associated with SLE susceptibility and with altered activation of the type I IFN pathway in SLE patients (Shrivastav and Niewold 2013). Unbiased genome-wide meta-analyses are recently increasing the number of loci, associated with a particular disease. For example, more than 100 such risk loci have been described for RA (e.g., Eyre et al. 2012). For sure, no single cause for the opposing effects mediated by type I IFNs in inflammatory processes can be identified - in Fig. 1 conditions contributing to either proor anti-inflammatory properties of type I IFNs are summarized.

\section{Cross-References}

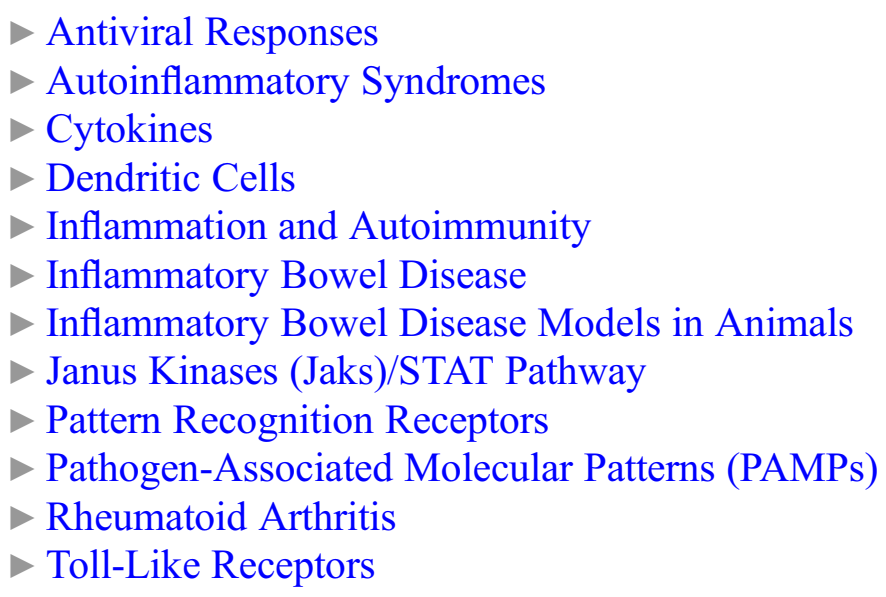




\section{References}

Afshar, M., Martinez, A. D., Gallo, R. L., \& Hata, T. R. (2013). Induction and exacerbation of psoriasis with Interferon-alpha therapy for hepatitis C: A review and analysis of 36 cases. Journal of the European Academy of Dermatology and Venereology, 27, 771-778.

Agrawal, H., Jacob, N., Carreras, E., Bajana, S., Putterman, C., Turner, S., Neas, B., Mathian, A., Koss, M. N., Stohl, W., Kovats, S., \& Jacob, C. O. (2009). Deficiency of type I IFN receptor in lupus-prone New Zealand mixed 2328 mice decreases dendritic cell numbers and activation and protects from disease. Journal of Immunology, 183, 6021-6029.

Axtell, R. C., Raman, C., \& Steinman, L. (2011). Interferon-beta exacerbates Th17-mediated inflammatory disease. Trends in Immunology, 32, 272-277.

Banchereau, J., \& Pascual, V. (2006). Type I interferon in systemic lupus erythematosus and other autoimmune diseases. Immunity, 25, 383-392.

Bissonnette, R., Papp, K., Maari, C., Yao, Y., Robbie, G., White, W. I., Le, C., \& White, B. (2010). A randomized, double-blind, placebo-controlled, phase I study of MEDI-545, an anti-interferon-alfa monoclonal antibody, in subjects with chronic psoriasis. Journal of the American Academy of Dermatology, 62, 427-436.

Bronson, P. G., Chaivorapol, C., Ortmann, W., Behrens, T. W., \& Graham, R. R. (2012). The genetics of type I interferon in systemic lupus erythematosus. Current Opinion in Immunology, 24, 530-537.

Crow, M. K. (2010). Type I interferon in organ-targeted autoimmune and inflammatory diseases. Arthritis Research \& Therapy, 12 (Suppl 1), S5.

de Weerd, N. A., Vivian, J. P., Nguyen, T. K., Mangan, N. E., Gould, J. A., Braniff, S. J., Zaker-Tabrizi, L., Fung, K. Y., Forster, S. C., Beddoe, T., Reid, H. H., Rossjohn, J., \& Hertzog, P. J. (2013). Structural basis of a unique interferon-beta signaling axis mediated via the receptor IFNAR1. Nature Immunology, 14, 901-907.

Eyre, S., Bowes, J., Diogo, D., Lee, A., Barton, A., Martin, P., Zhernakova, A., Stahl, E., Viatte, S., McAllister, K., Amos, C. I., Padyukov, L., Toes, R. E., Huizinga, T. W., Wijmenga, C., Trynka, G., Franke, L., Westra, H. J., Alfredsson, L., Hu, X., Sandor, C., de Bakker, P. I., Davila, S., Khor, C. C., Heng, K. K., Andrews, R., Edkins, S., Hunt, S. E., Langford, C., Symmons, D., Concannon, P., Onengut-Gumuscu, S., Rich, S. S., Deloukas, P., Gonzalez-Gay, M. A., Rodriguez-Rodriguez, L., Arlsetig, L., Martin, J., Rantapaa-Dahlqvist, S., Plenge, R. M., Raychaudhuri, S., Klareskog, L., Gregersen, P. K., \& Worthington, J. (2012). High-density genetic mapping identifies new susceptibility loci for rheumatoid arthritis. Nature Genetics, 44, 1336-1340.

Frenz, T., Waibler, Z., Hofmann, J., Hamdorf, M., Lantermann, M., Reizis, B., Tovey, M. G., Aichele, P., Sutter, G., \& Kalinke, U. (2010). Concomitant type I IFN receptor-triggering of T cells and of DC is required to promote maximal modified vaccinia virus Ankara-induced T-cell expansion. European Journal of Immunology, 40, 2769-2777.

Gonzalez-Navajas, J. M., Lee, J., David, M., \& Raz, E. (2012). Immunomodulatory functions of type I interferons. Nature Reviews Immunology, 12, 125-135.

Hida, S., Ogasawara, K., Sato, K., Abe, M., Takayanagi, H., Yokochi, T., Sato, T., Hirose, S., Shirai, T., Taki, S., \& Taniguchi, T. (2000). CD8(+) T cell-mediated skin disease in mice lacking IRF-2, the transcriptional attenuator of interferon-alpha/beta signaling. Immunity, 13, 643-655.

Kariuki, S. N., Franek, B. S., Kumar, A. A., Arrington, J., Mikolaitis, R. A., Utset, T. O., Jolly, M., Crow, M. K., Skol, A. D., \& Niewold, T. B. (2010). Trait-stratified genome-wide association study identifies novel and diverse genetic associations with serologic and cytokine phenotypes in systemic lupus erythematosus. Arthritis Research \& Therapy, 12, R151. 
Lauwerys, B. R., Ducreux, J., Houssiau, F. A. (2013). Type I interferon blockade in systemic lupus erythematosus: Where do we stand? Rheumatology. (Oxford), 53(8):1369-76.

Le Bon, A., Schiavoni, G., D’Agostino, G., Gresser, I., Belardelli, F., \& Tough, D. F. (2001). Type i interferons potently enhance humoral immunity and can promote isotype switching by stimulating dendritic cells in vivo. Immunity, 14, 461-470.

Marshak-Rothstein, A. (2006). Toll-like receptors in systemic autoimmune disease. Nature Reviews Immunology, 6, 823-835.

Matsuo, T., \& Takabatake, R. (2002). Multiple sclerosis-like disease secondary to alpha interferon. Ocular Immunology and Inflammation, 10, 299-304.

McCormack, P. L., \& Scott, L. J. (2004). Interferon-beta-1b: A review of its use in relapsing-remitting and secondary progressive multiple sclerosis. CNS Drugs, 18, 521-546.

Miller, J. C., Ma, Y., Bian, J., Sheehan, K. C., Zachary, J. F., Weis, J. H., Schreiber, R. D., \& Weis, J. J. (2008). A critical role for type I IFN in arthritis development following Borrelia burgdorferi infection of mice. Journal of Immunology, 181, 8492-8503.

Nestle, F. O., Conrad, C., Tun-Kyi, A., Homey, B., Gombert, M., Boyman, O., Burg, G., Liu, Y. J., \& Gilliet, M. (2005). Plasmacytoid predendritic cells initiate psoriasis through interferon-alpha production. Journal of Experimental Medicine, 202, 135-143.

Niewold, T. B., \& Swedler, W. I. (2005). Systemic lupus erythematosus arising during interferon-alpha therapy for cryoglobulinemic vasculitis associated with hepatitis C. Clinical Rheumatology, 24, 178-181.

Pitha, P. M. (2007). Interferon: The 50th anniversary. Berlin: Springer.

Rauch, I., Muller, M., \& Decker, T. (2013). The regulation of inflammation by interferons and their STATs. JAKSTAT, 2, e23820.

Rio, J., Nos, C., Tintore, M., Tellez, N., Galan, I., Pelayo, R., Comabella, M., \& Montalban, X. (2006). Defining the response to interferon-beta in relapsing-remitting multiple sclerosis patients. Annals of Neurology, 59, 344-352.

Shimizu, Y., Yokoyama, K., Misu, T., Takahashi, T., Fujihara, K., Kikuchi, S., Itoyama, Y., \& Iwata, M. (2008). Development of extensive brain lesions following interferon beta therapy in relapsing neuromyelitis optica and longitudinally extensive myelitis. Journal of Neurology, 255, 305-307.

Shrivastav, M., \& Niewold, T. B. (2013). Nucleic acid sensors and type I interferon production in systemic lupus erythematosus. Frontiers in Immunology, 4, 319.

van der Fits, L., van der Wel, L. I., Laman, J. D., Prens, E. P., \& Verschuren, M. C. (2004). In psoriasis lesional skin the type I interferon signaling pathway is activated, whereas interferon-alpha sensitivity is unaltered. The Journal of Investigative Dermatology, 122, 51-60.

Waibler, Z., Anzaghe, M., Frenz, T., Schwantes, A., Pohlmann, C., Ludwig, H., Palomo-Otero, M., Alcami, A., Sutter, G., \& Kalinke, U. (2009). Vaccinia virus-mediated inhibition of type I interferon responses is a multifactorial process involving the soluble type I interferon receptor B18 and intracellular components. Journal of Virology, 83, 1563-1571.

Yoneyama, M., \& Fujita, T. (2010). Recognition of viral nucleic acids in innate immunity. Reviews in Medical Virology, 20, 4-22.

Yong, V. W., Chabot, S., Stuve, O., \& Williams, G. (1998). Interferon beta in the treatment of multiple sclerosis: Mechanisms of action. Neurology, 51, 682-689. 